\title{
Subwavelength metamaterial engineering for silicon photonics
}

\author{
Robert Halir ${ }^{\mathrm{a}}$, Alejandro Ortega-Moñux ${ }^{\mathrm{a}}$, Jordi Soler-Penades ${ }^{\mathrm{b}}$, Jose M. Luque-González ${ }^{\mathrm{a}}$, \\ Darío Sarmiento-Merenguel ${ }^{\mathrm{a}}$, Alejandro Sánchez-Postigo ${ }^{\mathrm{a}}$, Gonzalo Wangüemert-Pérez ${ }^{\mathrm{a}}$, Jens \\ Schmid $^{\mathrm{c}}$, Dan-Xia Xuc ${ }^{\mathrm{c}}$, Siegfried Janz ${ }^{\mathrm{c}}$, Jean Lapointe ${ }^{\mathrm{c}}$, Íñigo Molina-Fernández ${ }^{\mathrm{a}}$, Milos \\ Nedeljkovic $^{\mathrm{b}}$, Goran Z. Mashanovich ${ }^{\mathrm{b}}$, and Pavel Cheben ${ }^{\mathrm{c}}$ \\ âUniversidad de Málaga, Dept. de Ingeniería de Comunicaciones, ETSI Telecomunicación, \\ 29071 Málaga, España \\ ${ }^{\mathrm{b}}$ Optoelectronics Research Centre, University of Southampton, Southampton SO17 1BJ, \\ United Kingdom \\ ${ }^{\mathrm{c} N a t i o n a l ~ R e s e a r c h ~ C o u n c i l ~ o f ~ C a n a d a, ~ O t t a w a, ~ O n t a r i o, ~ K 1 A ~ 0 R 6, ~ C a n a d a ~}$
}

\begin{abstract}
Waveguides structured at the subwavelength scale frustrate diffraction and behave as optical metamaterials with controllable refractive index. These structures have found widespread applications in silicon photonics, ranging from sub-decibel efficiency fibre-chip couplers to spectrometers and polarization rotators. Here, we briefly describe the design foundations for sub-wavelength waveguide devices, both in terms of analytic effective medium approximations, as well as through rigorous Floch-Bloquet mode simulation. We then focus on two novel structures that exemplify the use of subwavelength waveguides: mid-infrared waveguides and ultra-broadband beamsplitters.
\end{abstract}

Keywords: Silicon photonics, subwavelength structures, near infrared, mid infrared, ultra broadband devices

\section{INTRODUCTION}

The high lithographic resolution available in silicon photonics enables the realization of waveguides structured at the subwavelength scale, i.e. with dimensions small enough to frustrate diffraction effects. In a subwavelength waveguide, shown in Fig. 1, light propagates in the form of loss-less Bloch-Floquet modes. By adjusting the geometry of the subwavelength structure (e.g. its duty-cyle), lithographic control over the local refractive index is gained. ${ }^{1,2}$ This principle, which was well known in free space optics, has now found widespread applications in waveguide devices, ${ }^{3}$ with recent developments including wavelength filters, ${ }^{4,5}$ evanescent field sensors, ${ }^{6,7}$ and highly efficient fiber-to-chip couplers. ${ }^{8-12}$ Here we provide a short introduction on the general design of subwavelength waveguide devices (section 2), and then discuss two applications: suspended waveguides for the mid-infrared (section 3), and ultra-broadband couplers (section 4).

\section{DESIGNING WITH SUBWAVELENGTH STRUCTURES}

Several approaches can be used when modeling subwavelength waveguides. The simplest description is given in terms of the effective medium theory, ${ }^{1}$ which provides approximate values of the equivalent refractive index in terms of the fill-factor (or duty-cycle) of the structure. The effective medium approach is very useful to gain a qualitative understanding of the structure, but is only accurate in the deep sub-wavelength limit, i.e. when the pitch of the structure is much smaller than the operating wavelength. However, large dimensions (comparable to the wavelength in the medium) are often required to meet fabrication restrictions. In this situation analyzing the Floquet-Bloch modes of an equivalent 2D structure provides more reliable results. ${ }^{13}$ Since such $2 \mathrm{D}$ simulations are not time consuming they are well suited to explore new concepts and for initial device designs. However, the $2 \mathrm{D}$ approximation tends to significantly over-estimate the actual mode effective indices, and, more critically, underestimates leakage losses through the buried oxide to the silicon substrate. ${ }^{14}$ Therefore for a final design full 3D simulations, through either 3D FDTD or specialized methods, ${ }^{15}$ need be carried out. It is noted that accurate knowledge of the effective mode index is sufficient to control substrate leakage losses. ${ }^{14}$

Further author information:

Robert Halir: E-mail: robert.halir@ic.uma.es 


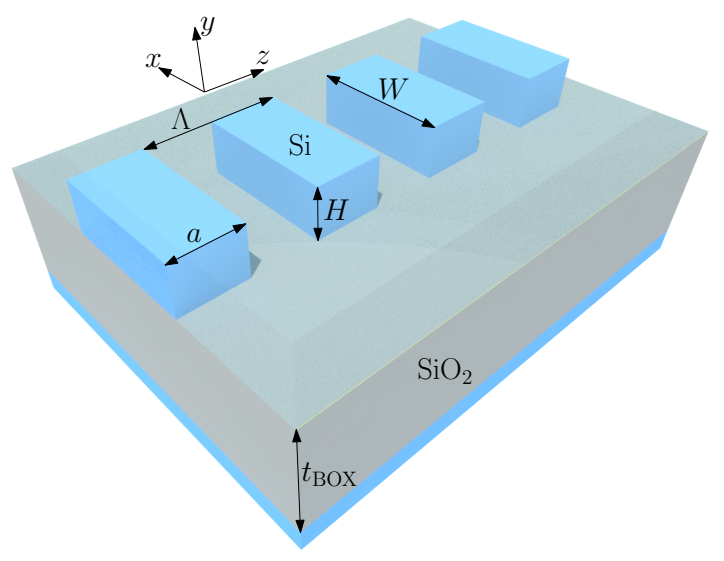

Figure 1. A subwavelength waveguide composed of alternating segments of silicon and the cladding material. The pitch $(\Lambda)$ is smaller than the wavelength of light propagating along the $z$-direction, which suppresses diffraction. The subwavelength waveguide thus behaves as a homogeneous medium with a refractive index that can be tuned by adjusting the duty-cycle, $a / \Lambda$, of the structure. At telecom wavelengths typical dimensions are: silicon thickness $H=220 \mathrm{~nm}$, buried oxide thickness $t_{\mathrm{BOX}}=3 \mu \mathrm{m}$ and waveguide width $W=500 \mathrm{~nm}$.

\section{MID-INFRARED WAVEGUIDES}

The mid-infrared wavelength range $(2 \mu \mathrm{m}-20 \mu \mathrm{m})$ is attracting increasing attention in integrated optics, because many compounds of interest, including explosives, can be detected through their "fingerprint" absorption spectra in this region. Silicon-on-insulator are not well suited for this wavelength range, because the silicon dioxide buried oxide layer becomes highly lossy beyond $4 \mu \mathrm{m}$. One way to leverage the advances of silicon photonics in the MidIR is to remove lossy oxide layer, resulting in suspended waveguides as shown in Fig. 2(a). ${ }^{16}$ In this structure the subwavelength structure in the cladding has a threefold functionality: i) it provides the necessary lateral index contrast to guide light in the waveguide core, ii) the openings allow for the penetration of hydrofluoric acid that etches away the lossy oxide layer under the waveguide, and iii) once the oxide is removed, the subwavelength structure provides mechanical support for the waveguide.
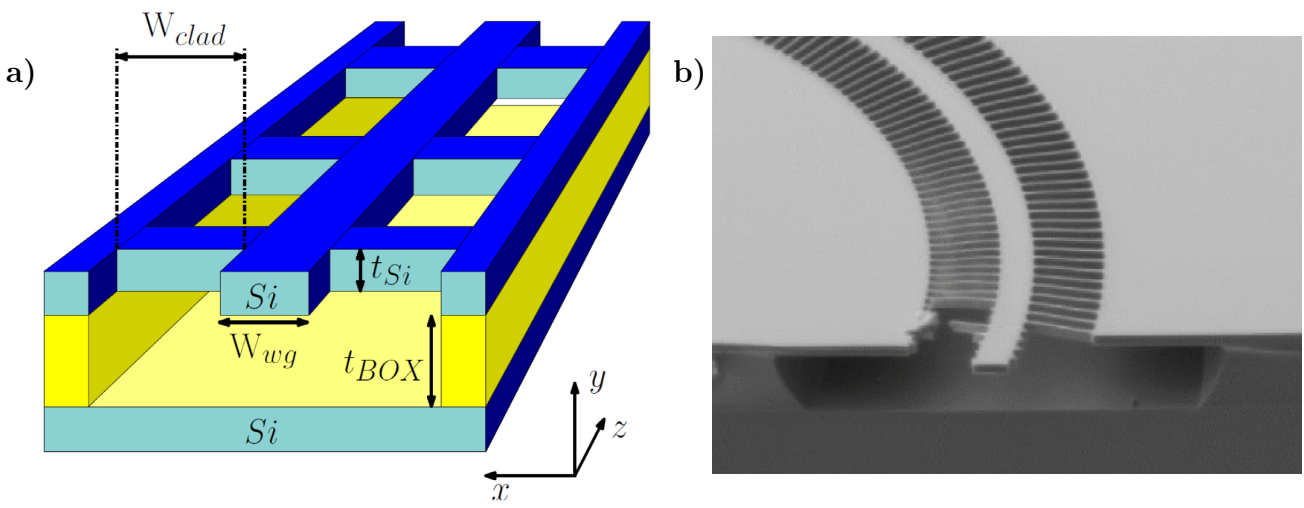

Figure 2. (a) A suspended silicon waveguide for mid-infrared applications. Light is guided in the silicon core, while the holes in the subwavelength cladding enable the penetration of hydrofluoric acid to etch away the buried oxide. (b) Scanning electron microscope image showing a cleaved curved waveguide that has been suspended.

Figure 2(b) shows a tilted scanning electron microscope image of a suspended waveguide (damage in the first periods is only due to the mechanical cleaving), for which measured propagation losses were $\sim 1 \mathrm{~dB} / \mathrm{cm}$, with curvature losses below $0.02 \mathrm{~dB} /$ bend around $\lambda=3.7 \mu \mathrm{m} .{ }^{17}$ 

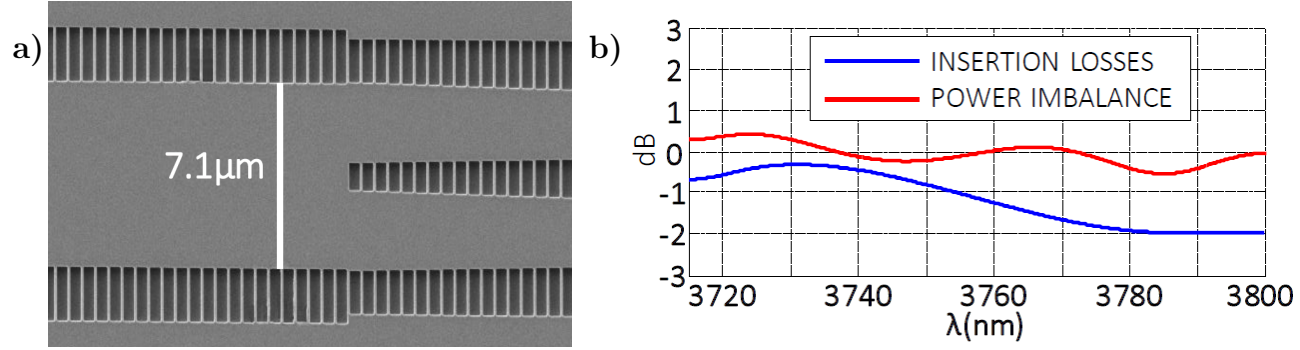

Figure 3. (a) Scanning electron microscope image of the output section of a fully suspended $2 \times 2$ MMI coupler. (b) Preliminary measurement results showing adequate performance of the MMI coupler around $\lambda=3.7 \mu \mathrm{m}$

Multimode interfernce couplers could also be successfully suspended, without producing collapse of these wider structures, as shown in Fig. 3(a). However, the hydrofluoric acid also slightly etched the silicon layer, which resulted in a device slightly thinner and narrower than designed, which explains the moderate insertion losses observed in the measurements shown in Fig. 3.

\section{BROADBAND WAVEGUIDE COUPLERS}

Integrated optical couplers cannot always match the performance and particularly the bandwidth of the bulkoptics counterparts. While adiabatic devices can offer impressive operating bandwidth, ${ }^{18}$ they usually sacrifice compactness to do so. Recently, several subwavelength devices have been proposed to achieve broadband operation. A directional coupler was proposed ${ }^{19}$ and demonstrated,${ }^{20}$ offering a bandwidth of approximately $100 \mathrm{~nm}$ at telecom wavelengths, where conventional directional couplers provide only of the order of $20 \mathrm{~nm}$. A compact adiabatic coupler based on subwavelength structures with a bandwidth of $130 \mathrm{~nm}$ was also recently presented. ${ }^{21}$

Multimode interference (MMI) couplers are well known to exhibit comparatively broad operational bandwidths, ${ }^{22}$ with $100 \mathrm{~nm}$ bandwidth attainable for a judiciously designed $2 \times 2$ device at telecom wavelengths. In an MMI coupler (see Fig. 4) light is launched through one of the input waveguides, and excites higher order modes in the central multimode region, which propagate and interfere forming images of the input field. These images are coupled to the output waveguides, achieving the desired $3 \mathrm{~dB}$ coupling. The position at which the images form thus dictates the length of the device, and is directly proportional to the beat-length of two lowest order modes of the multimode region: ${ }^{22}$

$$
L_{\pi}=\frac{\pi}{\beta_{1}-\beta_{2}} \approx \frac{4 W}{3 \lambda} n_{e q}
$$

Here, $W$ is the width of the multimode section, $\lambda$ is the free-space wavelength and $n_{e q}$ is the effective index of the multimode region. The operational bandwidth of these devices is ultimately limited by the wavelength dependence of the beat-length.

We recently showed that a subwavelength structure in the multimode region can be used to reduce the wavelength dependence of the beat-length, thereby enabling ultra-broadband couplers theoretically achieving bandwidths in excess of $500 \mathrm{~nm} .{ }^{23}$ This approach is based on the intrinsic anisotropy of the subwavelength structured multimode region, akin to that of a uni-axial crystal. As illustrated in Fig. 4(a) two waves polarized parallel and perpendicular to the silicon stripes experience different effective indices, $n_{x x}$ and $n_{z z}$. The wavelength dependence of $n_{x x}$ and $n_{z z}$ can be engineered by adjusting the pitch of the sub-wavelength structure; particularly $n_{x x}$ can become strongly dispersive in the vecinity of the Bragg wavelength of the structure. In the conventional 2D model of a MMI [see Fig. 4(b)] this effect can be modeled through an anisotropic meta-material represented by an effective index tensor $\mathbf{n}_{\mathbf{e q}}$. An approximate analytic expression for the beat-length, analogous to Eq. (1) can then be derived, yielding: ${ }^{23}$

$$
L_{\pi} \approx \frac{4 W}{3 \lambda} \frac{n_{z z}^{2}}{n_{x x}}
$$

From Eq. (2) we find that the beat-length is substantially decreased compared to the conventional device [Eq. (1)], because $n_{z z}$ is significantly smaller than $n_{x x}$ and $n_{e q}$. Furthermore, by adjusting the pitch of the 
a)

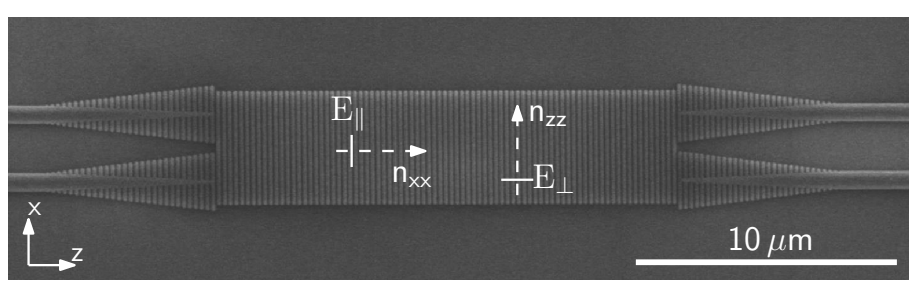

b)

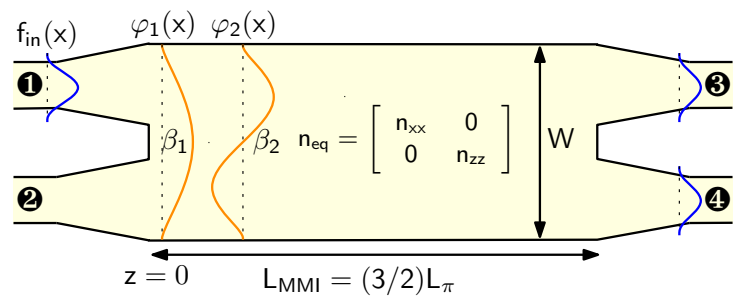

Figure 4. (a) Scanning electron microscope image of a multimode interference coupler (MMI) made of a subwavelength material that provides broadband operation. This is achieved through the characteristic anisotropy of the subwavelength structure in the central multimode region, analogous to a uni-axial crystal: the electric field parallel to the stripes $\left(E_{\|}\right)$ experiences a refractive index $n_{x x}$, whereas the perpendicular electric field $\left(E_{\perp}\right)$ experiences a refractive index $n_{z z}$. (b) Effective index model of the device shown in (a). The anisotropy is modeled through an effective index tensor $\mathbf{n}_{\mathbf{e q}}$, providing analytic expressions for the design of the device.

subwavelength structure, the wavelength dependence of $n_{x x}$ to minimize the overall wavelength dependence of $L_{\pi}$, which provides the desired broadband behavior.

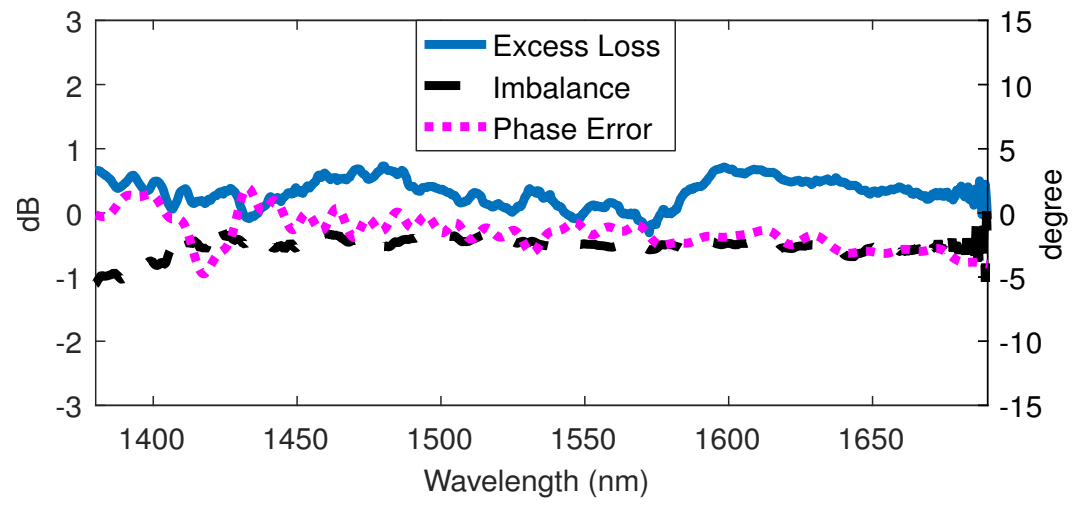

Figure 5. Measured performance of the fabricated broadband MMI, showing high performance over a bandwidth of more than $300 \mathrm{~nm}$ at telecom wavelengths.

3D FDTD simulations show that using this approach a $2 \times 2$ coupler with a bandwidth in excess of $500 \mathrm{~nm}$ can be obtained. Measurements of a fabricated device, shown in Fig. 5, reveal virtually perfect device performance in a bandwidth of over $300 \mathrm{~nm}$, with excess losses and imbalance below $1 \mathrm{~dB}$ and a deviation from the ideal $90^{\circ}$ phase shift between the two outputs of less than $5^{\circ}$.

\section{CONCLUSIONS}

Waveguide subwavelength structures are quickly becoming a versatile design tool in the silicon photonic platform. The unique ability to engineer refractive index and even anisotropy lithographically is giving rise to a wide array of devices with unique characteristics, such as ultra-broad bandwidth or low loss guiding in the mid-IR. We are confident that this trend will continue and that the future holds exciting design opportunities in this field. 


\section{ACKNOWLEDGMENTS}

We acknowledge funding from the Ministerio de Economía y Competitividad, Programa Estatal de Investigación, Desarrollo e Innovación Orientada a los Retos de la Sociedad (cofinanciado FEDER), Proyecto TEC2013-46917C2-1-R, Proyecto TEC2016-80718-R, and the Universidad de Málaga.

\section{REFERENCES}

[1] Rytov, S. M., "The electromagnetic properties of finely layered medium," Sov. Phys. JETP 2, 466-475 (1956).

[2] Cheben, P., Bock, P. J., Schmid, J. H., Lapointe, J., Janz, S., Xu, D.-X., Densmore, A., Delâge, A., Lamontagne, B., and Hall, T. J., "Refractive index engineering with subwavelength gratings for efficient microphotonic couplers and planar waveguide multiplexers," Opt. Lett. 35(15), 2526-2528 (2010).

[3] Halir, R., Bock, P. J., Cheben, P., Ortega-Moñux, A., Alonso-Ramos, C., Schmid, J. H., Lapointe, J., Xu, D.-X., Wangüemert-Pérez, J. G., Molina-Fernández, Í., et al., "Waveguide sub-wavelength structures: a review of principles and applications," Laser Photonics Rev. 9(1), 25-49 (2015).

[4] Wang, J., Glesk, I., and Chen, L. R., "Subwavelength grating filtering devices," Opt. Express 22, 1533515345 (Jun 2014).

[5] Donzella, V., Sherwali, A., Flueckiger, J., Grist, S. M., Fard, S. T., and Chrostowski, L., "Design and fabrication of soi micro-ring resonators based on sub-wavelength grating waveguides," Opt. Express 23, 4791-4803 (Feb 2015).

[6] Wangüemert-Pérez, J. G., Cheben, P., nux, A. O.-M., Alonso-Ramos, C., Pérez-Galacho, D., Halir, R., nigo Molina-Fernández, I., Xu, D.-X., and Schmid, J. H., "Evanescent field waveguide sensing with subwavelength grating structures in silicon-on-insulator," Opt. Lett. 39, 4442-4445 (Aug 2014).

[7] Flueckiger, J., Schmidt, S., Donzella, V., Sherwali, A., Ratner, D. M., Chrostowski, L., and Cheung, K. C., "Sub-wavelength grating for enhanced ring resonator biosensor," Opt. Express 24, 15672-15686 (Jul 2016).

[8] Cheben, P., Xu, D.-X., Janz, S., and Densmore, A., "Subwavelength waveguide grating for mode conversion and light coupling in integrated optics," Opt. Express 14(11), 4695-4702 (2006).

[9] Wang, Y., Yun, H., Lu, Z., Bojko, R., Shi, W., Wang, X., Flueckiger, J., Zhang, F., Caverley, M., Jaeger, N. A. F., and Chrostowski, L., "Apodized focusing fully etched subwavelength grating couplers," IEEE Photonics Journal 7, 1-10 (June 2015).

[10] Cheben, P., Schmid, J. H., Wang, S., Xu, D.-X., Vachon, M., Janz, S., Lapointe, J., Painchaud, Y., and Picard, M.-J., "Broadband polarization independent nanophotonic coupler for silicon waveguides with ultrahigh efficiency," Opt. Express 23(17), 22553-22563 (2015).

[11] Benedikovic, D., Alonso-Ramos, C., Cheben, P., Schmid, J. H., Wang, S., Halir, R., nux, A. O.-M., Xu, D.-X., Vivien, L., Lapointe, J., Janz, S., and Dado, M., "Single-etch subwavelength engineered fiber-chip grating couplers for 1.3 $\mu \mathrm{m}$ datacom wavelength band," Opt. Express 24, 12893-12904 (Jun 2016).

[12] Sánchez-Postigo, A., Wangüemert-Pérez, J. G., Luque-González, J. M., Molina-Fernández, I., Cheben, P., Alonso-Ramos, C. A., Halir, R., Schmid, J. H., and Ortega-Moñux, A., "Broadband fiber-chip zero-order surface grating coupler with 0.4db efficiency," Opt. Lett. 41, 3013-3016 (Jul 2016).

[13] Zavargo-Peche, L., Ortega-Moñux, A., Wangüemert-Pérez, J., and Molina-Fernández, I., "Fourier based combined techniques to design novel sub-wavelength optical integrated devices," Prog. Electromagn. Res. 123, 447-465 (2012).

[14] Sarmiento-Merenguel, J. D., nux, A. O.-M., Fédéli, J.-M., Wangüemert-Pérez, J. G., Alonso-Ramos, C., Durán-Valdeiglesias, E., Cheben, P., nigo Molina-Fernández, I., and Halir, R., "Controlling leakage losses in subwavelength grating silicon metamaterial waveguides," Opt. Lett. 41, 3443-3446 (Aug 2016).

[15] Čtyrokỳ, J., "3-d bidirectional propagation algorithm based on fourier series," J. Lightwave Technol. 30(23), 3699-3708 (2012).

[16] Penadés, J. S., Alonso-Ramos, C., Khokhar, A. Z., Nedeljkovic, M., Boodhoo, L. A., Ortega-Moñux, A., Molina-Fernández, I., Cheben, P., and Mashanovich, G. Z., "Suspended soi waveguide with sub-wavelength grating cladding for mid-infrared," Opt. Lett. 39, 5661-5664 (Oct 2014). 
[17] Penades, J. S., Ortega-Moñux, A., Nedeljkovic, M., Wangüemert-Pérez, J. G., Halir, R., Khokhar, A. Z., Alonso-Ramos, C., Qu, Z., Molina-Fernández, I., Cheben, P., and Mashanovich, G. Z., "Suspended silicon mid-infrared waveguide devices with subwavelength grating metamaterial cladding," Opt. Express 24, 2290822916 (Oct 2016).

[18] Solehmainen, K., Kapulainen, M., Harjanne, M., and Aalto, T., "Adiabatic and multimode interference couplers on silicon-on-insulator," IEEE Photonics Technol. Lett. 18(21), 2287-2289 (2006).

[19] Halir, R., Maese-Novo, A., Ortega-Moñux, A., Molina-Fernández, I., Wangüemert-Pérez, J., Cheben, P., Xu, D.-X., Schmid, J., and Janz, S., "Colorless directional coupler with dispersion engineered sub-wavelength structure," Opt. Express 20(12), 13470-13477 (2012).

[20] Wang, Y., Lu, Z., Ma, M., Yun, H., Zhang, F., Jaeger, N., and Chrostowski, L., "Compact broadband directional couplers using sub-wavelength gratings," IEEE Photon. J. (2016).

[21] Yun, H., Wang, Y., Zhang, F., Lu, Z., Lin, S., Chrostowski, L., and Jaeger, N. A., "Broadband $2 \times 2$ adiabatic $3 \mathrm{~dB}$ coupler using silicon-on-insulator sub-wavelength grating waveguides," Opt. Lett. 41(13), 3041-3044 (2016).

[22] Soldano, L. B. and Pennings, E. C., "Optical multi-mode interference devices based on self-imaging: principles and applications," J. Lightwave Technol. 13(4), 615-627 (1995).

[23] Halir, R., Cheben, P., Luque-González, J., Sarmiento-Merenguel, J., Schmid, J. H., Wangüemert-Pérez, G., Xu, D.-X., Wang, S., Ortega-Moñux, A., and Molina-Fernández, I., "Ultra-broadband nanophotonic beamsplitter using an anisotropic sub-wavelength metamaterial," Laser Photonics Rev. 10, 1039-1046 (2016). 